\title{
INFLUÊNCIA DAS COMPOSIÇÕES QUÍMICA E MINERALÓGICA DE CINZAS DE MISTURAS DE CARVÕES PARA PCI NO COMPORTAMENTO DE FUSIBILIDADE E VISCOSIDADE A ALTAS TEMPERATURAS
}

Maria Luisa Formoso Ghiggi । Eduardo Osório ${ }^{2}$ Antonio Cezar Faria Vilela ${ }^{3}$ Ricardo Jose Tauffer Barros ${ }^{4}$ Roberto da Cruz Junior ${ }^{5}$

\section{Resumo}

As causas da deterioração da permeabilidade da carga no alto-forno sob taxas altas de injeção de carvão pulverizado ainda não são bem conhecidas. Sabe-se na prática operacional que variações na qualidade das cinzas dos carvões injetados podem afetar a estabilidade do forno. Trabalho anterior analisou o comportamento de cinzas de carvões a altas temperaturas em relação às suas composições químicas e mineralógicas. O objetivo deste trabalho foi avaliar o mesmo comportamento em misturas de carvões. Amostras de quatro carvões e sete misturas foram queimadas entre $800-850^{\circ} \mathrm{C}$ e suas cinzas analisadas por fluorescência e difração de raios $X$ e por testes de fusibilidade e viscosidade. Cinzas com teores mais altos de $\mathrm{Si}$ e $\mathrm{Al}$ apresentaram alta temperatura de fluidez e maior viscosidade a $1.500^{\circ} \mathrm{C}$, devido à grande proporção de mulita e à baixa basicidade. Cinzas com teores significativos de $\mathrm{Fe}, \mathrm{Ca}$ e $\mathrm{S}$ apresentaram temperaturas de fluidez e viscosidade mais baixas, em função da transformação de mulita em anortita e/ou da mais alta basicidade.

Palavras-chave: Injeção de carvão pulverizado; Cinzas; Carvão; Fusibilidade; Viscosidade.

\section{INFLUENCE OF THE CHEMICAL AND MINERALOGYCAL ASH COMPOSITION OF COAL BLENDS FOR PCI IN THE BEHAVIOR OF FUSIBILITY AND VISCOSITY AT HIGH TEMPERATURES}

\begin{abstract}
Some aspects of permeability deterioration in blast furnace process at high rates of pulverized coal injection are not well known yet. At operational practice variations in the ash quality of injected coals can affect the furnace performance. Previous work has investigated the behavior of individual coals ash at high temperatures in relation to the chemical and mineralogical compositions. This work aimed to assess this behavior for coal blends. Four coals and seven blends were selected and ashed at $800-850^{\circ} \mathrm{C}$. The samples were analyzed by X-ray fluorescence and X-ray diffraction techniques and ASTM ash fusion (AFT) and viscosity tests were performed. Ash containing high contents of Si and Al showed higher fusion temperatures and higher viscosity at $1500^{\circ} \mathrm{C}$, due mainly to the higher content of Mullite and to the low basicity, while those presenting significant contents of $\mathrm{Fe}, \mathrm{Ca}$ and $\mathrm{S}$ had lower fusion temperatures and lower viscosity, due to the higher content of Anorthite and/or to the higher basicity.
\end{abstract}

Key words: Pulverized coal injection; Ash; Coal; Fusibility; Viscosity.

\footnotetext{
'Engenheira Metalúrgica, Aluna do Programa de Pós-Graduação em Engenharia de Minas, Metalúrgica e de Materiais, Universidade Federal do Rio Grande do Sul - UFRGS, Av. Bento Gonçalves, 9500, Cep 91501-970, Porto Alegre, RS, Brasil. E-mail: luisa.ghiggi@ufrgs.br

${ }^{2}$ Dr. Ing. Prof. do Departamento de Metalurgia da Escola de Engenharia da Universidade Federal do Rio Grande do Sul - UFRGS, Av. Bento Gonçalves, 9500, Cep 9150I-970, Porto Alegre, RS, Brasil. E-mail: vilela@ufrgs.br

${ }^{3}$ Doutor em Engenharia, Prof. do Departamento de Metalurgia da Escola de Engenharia, Universidade Federal do Rio Grande do Sul - UFRGS,

Av. Bento Gonçalves, 9500, Cep 91501-970, Porto Alegre, RS, Brasil.E-mail: eosorio@ufrgs.br

${ }^{4}$ Engenheiro Metalurgista, Especialista em Alto-Forno ArcelorMittal Tubarão, Av. Brigadeiro Eduardo Gomes, 930, Cep 29I63-970, Serra, ES, Brasil. E-mail: ricardo.tauffer@arcelormittal.com

${ }^{5}$ Engenheiro Metalurgista, Especialista em Alto-Forno ArcelorMittal Tubarão, Av. Brigadeiro Eduardo Gomes, 930, Cep 29I63-970, Serra, ES, Brasil. E-mail: roberto.dacruz@arcelormittal.com.
} 


\section{INTRODUÇÃO}

Nas duas últimas décadas, a maioria dos processos de produção de ferro primário em altos fornos tem utilizado a tecnologia de injeção de carvão pulverizado $(\mathrm{PCl})$ em função dos benefícios econômicos, operacionais e ambientais atingidos.

Os principais fatores que afetam o desempenho da injeção de carvão no alto-forno são as propriedades do carvão e as condições de combustão, moagem, transporte e injeção do carvão.(I) Uma vez que a tecnologia de injeção está bem consolidada, o decréscimo na permeabilidade da carga na região inferior do alto-forno geralmente está associado a uma má combustão dos carvões na zona das ventaneiras e à geração de excesso de finos de "char". Entretanto, quando os carvões apresentam uma combustão adequada para o processo e a permeabilidade cai, buscam-se as causas nas variações da composição química das cinzas dos carvões. Os mecanismos pelos quais a qualidade das cinzas afeta a estabilidade operacional do alto forno ainda não estão bem estabelecidos. Ichida et al. ${ }^{(2)}$ sugeriram, que as cinzas do carvão pulverizado em altas taxas de injeção podem modificar as características da escória gotejante acima das ventaneiras, promovendo a formação do birdsnest e deteriorando a permeabilidade na zona do homem-morto. Para evitar a excessiva basicidade da escória da região da rampa devido à injeção de carvão pulverizado, Wikström et al. ${ }^{(3)}$ utilizaram a co-injeção de carvão pulverizado com pó de coletor de alto-forno e escória de aciaria (conversor LD) num alto forno experimental. Esses autores observaram que a redução da acidez da escória de ventaneira, evitava a formação de birdsnest e deixava a operação mais suave e estável.

Trabalhos anteriores ${ }^{(4,5)}$ analisaram 0 comportamento de cinzas de carvões em temperaturas altas, através de testes de fusibilidade e viscosidade e sua relação com as composições química e mineralógica. Como normalmente se usam misturas de carvões alto (AV) e baixo voláteis (BV) no processo de injeção de carvão para obter a combustão e a taxa de substituição coque/carvão desejadas, o objetivo deste trabalho é avaliar o comportamento de fusibilidade e de viscosidade a altas temperaturas de cinzas de misturas de carvões em relação às composições química e mineralógica, para dar suporte à seleção de carvões para $\mathrm{PCl}$.

\section{MATERIAIS E MÉTODOS}

Foram selecionados quatro carvões usados no processo de $\mathrm{PCl}$, denominados $\mathrm{A}, \mathrm{B}, \mathrm{C}$ e $\mathrm{D}$. Desses carvões foram preparadas três misturas binárias $A D$ e 4 misturas ternárias $A B C$, visando formar misturas que tivessem uma qualidade adequada para $\circ$ processo de combustão no alto-forno. Na Tabela I encontram-se a análise imediata dos carvões (ASTM D7582-10(6)), a de enxofre total (ASTM D4239-10(7)) e as proporções dos carvões nas misturas.

Os carvões e misturas foram queimados entre $800^{\circ} \mathrm{C}-850^{\circ} \mathrm{C}$, em forno mufla, e suas cinzas preparadas para análise química por espectrometria de fluorescência de raios $X(F R X)$, análise mineralógica por difração de raios $X(D R X)$, testes de fusibilidade e testes em viscosímetro rotacional de $1.600^{\circ} \mathrm{C}$.

$\mathrm{Na}$ análise química das cinzas por FRX (ASTM D4326-04(8) foram determinados somente os principais elementos, descritos na forma de óxidos, $\mathrm{SiO}_{2}$, $\mathrm{Al}_{2} \mathrm{O}_{3}, \mathrm{Fe}_{2} \mathrm{O}_{3}, \mathrm{CaO}, \mathrm{MgO}, \mathrm{Na}_{2} \mathrm{O}, \mathrm{K}_{2} \mathrm{O}, \mathrm{TiO}_{2}, \mathrm{P}_{2} \mathrm{O}_{5}$ e o teor de $\mathrm{SO}_{3}$ (ASTM D50 I6-08e I ${ }^{(9)}$ ).

Para a discussão dos resultados, calculou-se a razão entre os teores de sílica e alumina e a basicidade, pela razão entre o somatório da fração molar dos óxidos básicos $\left(\mathrm{Fe}_{2} \mathrm{O}_{3}, \mathrm{CaO}, \mathrm{MgO}, \mathrm{K}_{2} \mathrm{O}, \mathrm{Na}_{2} \mathrm{O}\right)$ e o somatório da fração molar dos óxidos ácidos $\left(\mathrm{Al}_{2} \mathrm{O}_{3}, \mathrm{SiO}_{2}\right)$ nas cinzas, considerando o $\mathrm{Fe}^{3+}$ agindo como óxido básico devido a seu comportamento anfótero. ${ }^{(10)}$

A técnica utilizada para DRX, difratômetro com monocromador de $\mathrm{C}$, usando radiação $\mathrm{Cu} \mathrm{K} \alpha$, permite identificar os minerais e as fases cristalinas presentes acima de $5 \%$ em volume. Para essa análise, as amostras de cinzas foram reduzidas a -325 mesh. A análise mineralógica foi realizada nos carvões in natura, nas cinzas dos carvões e misturas queimadas entre $800^{\circ} \mathrm{C}-850^{\circ} \mathrm{C}$ e nas cinzas aquecidas até $1.240^{\circ} \mathrm{C}$, resfriadas ao ar.

Nos testes de fusibilidade, as amostras de cinzas, preparadas na forma de um cone, foram aquecidas a uma taxa de $8 \pm 3^{\circ} \mathrm{C} / \mathrm{min}$, em atmosfera de ar, até atingir a fluidez (ASTM DI857-04(11)). Foram determinadas as temperaturas características de fusibilidade dos cones (Figura I):

- IT - temperatura de início de deformação (arredondamento) da ponta do cone;

- ST - temperatura de esfera: altura $\mathrm{H}=$ largura L da base;

Tabela I. Análise química dos carvões e composição das misturas em base seca (\% em massa)

\begin{tabular}{ccccccccccc}
\hline Amostra & $\mathbf{C z}$ & MV & Stotal & ABCI & ABC2 & ABC3 & ABC4 & ADI & AD2 & AD3 \\
\hline A & 7,99 & 37,03 & 0,83 & 50 & 40 & 30 & 20 & 60 & 50 & 40 \\
B & 7,39 & 37,06 & 1,73 & 10 & 20 & 30 & 40 & - & - & - \\
C & 9,16 & 12,40 & 0,26 & 40 & 40 & 40 & 40 & - & - & - \\
D & 5,38 & 19,67 & 0,77 & - & - & - & - & 40 & 50 & 60 \\
\hline
\end{tabular}

Cz: Cinzas; MV: Matéria volátil. 
- HT - temperatura de semi-esfera: $\mathrm{H}=\mathrm{L} / 2$; e

- FT - temperatura de fluidez: $\mathrm{H} \leq \mathrm{I}, 8 \mathrm{~mm}$.

Foram testados 5 cones por amostra, sendo o erro na temperatura de $\pm 5^{\circ} \mathrm{C}$ e repetibilidade nos testes de $\pm 30^{\circ} \mathrm{C}$.

Os testes de viscosidade foram executados num viscosímetro rotativo, cujo eixo utiliza velocidade angular constante para medir a viscosidade a diferentes temperaturas. Após calibração do viscosímetro, uma amostra de cerca de $300 \mathrm{~g}$ de cinzas foi aquecida num cadinho de platina até $1.600^{\circ} \mathrm{C}$, em atmosfera de ar, para a fusão e mantida por um tempo pré-determinado até atingir o equilíbrio térmico. As viscosidades foram registradas em função da velocidade da haste e da temperatura do líquido no resfriamento da amostra (norma A - ASTM C-965(12)).

Além dos testes planejados, foram realizados testes de aquecimento das cinzas em temperatura mais alta, com o objetivo de verificar as fases presentes próximas a menor temperatura inicial de deformação do cone. Os testes foram realizados no forno do microscópio de aquecimento, usado para testes de fusibilidade, conforme norma DIN 51730.(13) Para os testes de aquecimento propriamente ditos, amostras de $90 \mathrm{mg}$ foram levemente compactadas num cadinho de $\mathrm{Al}_{2} \mathrm{O}_{3}$. Cada amostra foi colocada sobre o termopar tipo " $\mathrm{S}$ ", protegido com uma camisa de $\mathrm{Al}_{2} \mathrm{O}_{3}$, no centro do forno, e aquecida até $1.240 \pm 10^{\circ} \mathrm{C}$, numa taxa de $8 \pm 3^{\circ} \mathrm{C} / \mathrm{min}$, em atmosfera de ar. Após a sinterização, a amostra foi retirada em menos de $5 \mathrm{~s}$, junto com o termopar, pela saída lateral, e resfriada ao ar. A variação da temperatura da amostra do centro até a saída do forno foi de $20^{\circ} \mathrm{C}$ e a taxa de resfriamento do termopar abaixo da amostra de $300^{\circ} \mathrm{C} / \mathrm{min}$. As amostras de cinzas sinterizadas foram moídas e analisadas por DRX.

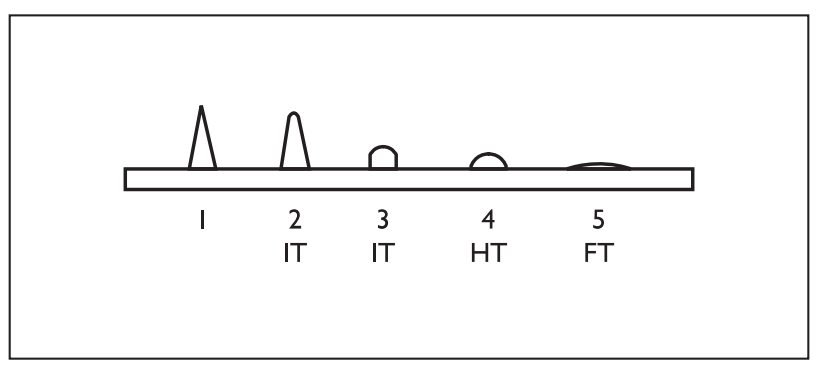

Figura I. Temperaturas características dos testes de fusibilidade ASTM.

\section{RESULTADOS E DISCUSSÃO}

\section{I Análise Química das Cinzas}

A Tabela 2 apresenta os resultados da análise química das cinzas dos carvões e também a razão sílica/ alumina e a basicidade dessas cinzas. Como nem todos os elementos se encontram na forma óxida, a avaliação foi realizada com base nos elementos contidos nas cinzas.

Verifica-se nessa tabela que as composições químicas das cinzas dos carvões são bem distintas. Os elementos de maior concentração em todas as cinzas são $\mathrm{Si}$ e $\mathrm{Al}$, sendo as cinzas " $\mathrm{A}$ " as mais ricas em $\mathrm{Si}$ e as cinzas "C", as mais ricas em $\mathrm{Al}$. $\mathrm{A}$ razão $\mathrm{SiO}_{2} / \mathrm{Al}_{2} \mathrm{O}_{3}$ dá uma idéia da maior ou menor proporção de silicatos e aluminossilicatos presentes nas cinzas. Esta relação é maior para as cinzas "A" e "B" e menor para as cinzas "C" e "D". Quanto aos elementos minoritários - Fe, Ca e S, a amostra "B" tem o teor de Fe mais alto, seguida pela amostra "D", que se distingue por ser mais rica em Ca e também em S. Mesmo em pequenas quantidades, os elementos minoritários podem ser importantes, como $\mathrm{K}$ e $\mathrm{Na}$ que formam fases de mais baixo ponto de fusão e $\mathrm{Mg}$ que pode reduzir a viscosidade das cinzas líquidas ou $\mathrm{Ti}$ e $\mathrm{P}$, que tendem a formar fases de alto ponto de fusão. ${ }^{(14)}$ Quanto à basicidade, observa-se que as cinzas mais ácidas correspondem à amostra "A" e as mais básicas à amostra "D".

$\mathrm{Na}$ Tabela 3, encontram-se as análises químicas das cinzas das misturas de carvões, bem como a razão sílica/ alumina e a basicidade dessas cinzas. Nas misturas "ABC", observa-se que o teor de Si tende a decrescer de "ABCI" para "ABC4", enquanto que o teor de Al é praticamente constante, o que causa um decréscimo da razão sílica/ alumina de I,89 para I,8I. Entre os outros elementos, destacam-se o teor de $\mathrm{Fe}$, que aumenta de "ABCI" para "ABC4", e os teores de $\mathrm{Mg}, \mathrm{K}$ e S, que decrescem. Em função da pequena diferença de composição química nessas misturas, a basicidade varia pouco. Nas misturas "AD", verifica-se de "ADI" para "AD3", uma redução no teor de Si e na razão sílica/alumina em função da menor participação do carvão A. Entre os outros elementos, há uma leve tendência de aumento no teor de $\mathrm{Fe}$ e um aumento significativo nos teores de $\mathrm{Ca}$ e $\mathrm{S}$. Comparando as duas séries de misturas, constata-se que as misturas "ABC" têm maior teor de $\mathrm{Al}$ e menor basicidade que as misturas "AD".

Tabela 2. Análise química por FRX, razão sílica-alumina e basicidade das cinzas dos carvões individuais

\begin{tabular}{ccccccccccccc}
\hline $\mathbf{C z}$ & $\begin{array}{c}\mathrm{SiO}_{2} \\
\%\end{array}$ & $\begin{array}{c}\mathbf{A l}_{2} \mathbf{O}_{3} \\
\%\end{array}$ & $\begin{array}{c}\mathbf{F e}_{2} \mathbf{O}_{3} \\
\%\end{array}$ & $\begin{array}{c}\mathbf{C a O} \\
\%\end{array}$ & $\begin{array}{c}\mathbf{M g O} \\
\%\end{array}$ & $\begin{array}{c}\mathbf{N a}_{2} \mathbf{O} \\
\%\end{array}$ & $\begin{array}{c}\mathbf{K}_{2} \mathbf{O} \\
\%\end{array}$ & $\begin{array}{c}\mathbf{T i O}_{2} \\
\%\end{array}$ & $\begin{array}{c}\mathbf{P}_{2} \mathbf{O}_{5} \\
\%\end{array}$ & $\begin{array}{c}\mathbf{S O}_{3} \\
\%\end{array}$ & $\begin{array}{c}\mathbf{S i O}_{2} / \\
\mathbf{A l}_{2} \mathbf{O}_{3}\end{array}$ & $\begin{array}{c}\mathbf{B}_{\%} \\
\%\end{array}$ \\
\hline $\mathrm{A}$ & 58,03 & 23,90 & 8,00 & 2,30 & 2,02 & 0,22 & 1,94 & 0,99 & $<0,03$ & $\mathrm{I}, 65$ & 2,42 & 0,14 \\
$\mathrm{~B}$ & 49,22 & 23,70 & 17,89 & 2,71 & 0,61 & 0,26 & 1,87 & 1,20 & 0,28 & $\mathrm{I}, 15$ & 2,07 & 0,18 \\
$\mathrm{C}$ & 42,75 & 32,53 & 6,19 & 6,31 & 1,40 & 0,22 & 1,74 & 1,76 & 2,68 & 2,57 & 1,31 & 0,20 \\
$\mathrm{D}$ & 32,29 & 21,07 & 12,51 & 12,51 & 1,89 & 0,22 & 1,37 & 1,16 & $<0,03$ & 14,66 & 1,53 & 0,49 \\
\hline
\end{tabular}

$\mathrm{B}=$ Basicidade $; \mathrm{C} z=$ Cinzas. 


\subsection{Análise Mineralógica dos Carvões e das Cinzas por Difração de Raios $\mathbf{X}$}

\subsection{Principais minerais nos carvões}

A Figura 2 apresenta os difratogramas de raios $X$ das amostras dos carvões. Devido ao efeito de diluição na matéria orgânica, ${ }^{(5)}$ aparecem somente os picos das fases mais importantes. No carvão "A", os principais minerais são caolinita e quartzo, sendo o pico mais importante do quartzo (IOI) de grande intensidade. No carvão "B", aparecem os picos correspondentes aos argilominerais caolinita e ilita e aos minerais quartzo e pirita. Observa-se que o pico principal do quartzo no carvão "B" é menos intenso que no carvão " $A$ ", sugerindo uma menor quantidade desse mineral no carvão "B". O carvão "B" possui o maior teor de pirita entre os carvões, de acordo com o maior teor de $\mathrm{S}$ encontrado em sua análise química na Tabela I. No carvão "C", os principais minerais são caolinita, calcita e fluorapatita. Destacam-se a ausência do quartzo e a presença de minerais ricos em $\mathrm{Ca}$. De acordo com a análise química das cinzas, Tabela 2, o carvão "C" é rico em Al e a razão sílica/alumina é a menor entre os carvões. Isto permite concluir que o carvão "C" possui o maior teor de caolinita entre os demais. No carvão "D", aparecem os minerais caolinita, calcita e quartzo, sendo o pico do quartzo de menor intensidade que aqueles dos carvões "A" e "B".

Tabela 3. Análise química por FRX, razão sílica/alumina e basicidade das cinzas das misturas de carvões

\begin{tabular}{|c|c|c|c|c|c|c|c|c|c|c|c|c|}
\hline $\mathrm{Cz}$ & $\begin{array}{c}\mathrm{SiO}_{2} \\
\%\end{array}$ & $\begin{array}{c}\mathrm{Al}_{2} \mathbf{O}_{3} \\
\%\end{array}$ & $\begin{array}{c}\mathrm{Fe}_{2} \mathbf{O}_{3} \\
\%\end{array}$ & $\begin{array}{c}\mathrm{CaO} \\
\%\end{array}$ & $\begin{array}{c}\text { MgO } \\
\%\end{array}$ & $\begin{array}{c}\mathrm{Na}_{2} \mathbf{O} \\
\%\end{array}$ & $\begin{array}{c}\mathbf{K}_{2} \mathbf{O} \\
\%\end{array}$ & $\begin{array}{c}\mathrm{TiO}_{2} \\
\%\end{array}$ & $\begin{array}{c}\mathbf{P}_{2} \mathbf{O}_{5} \\
\%\end{array}$ & $\begin{array}{c}\mathbf{S O}_{3} \\
\%\end{array}$ & $\begin{array}{l}\mathrm{SiO}_{2} / \\
\mathrm{Al}_{2} \mathbf{O}_{3}\end{array}$ & $\begin{array}{l}\text { B } \\
\%\end{array}$ \\
\hline $\mathrm{ABCl}$ & 51,26 & 27,12 & 8,47 & 3,85 & 1,62 & 0,24 & 1,80 & 1,30 & $\mathrm{I}, 05$ & 1,94 & 1,89 & 0,17 \\
\hline$A B C 2$ & 50,35 & 27,05 & 9,60 & 3,88 & $\mathrm{I}, 47$ & 0,23 & $\mathrm{I}, 74$ & 1,32 & $\mathrm{I}, 07$ & I,88 & 1,86 & 0,17 \\
\hline $\mathrm{ABC} 3$ & 49,47 & 26,98 & 10,69 & 3,90 & 1,32 & 0,23 & 1,67 & $\mathrm{I}, 34$ & 1,08 & 1,82 & 1,83 & 0,18 \\
\hline $\mathrm{ABC} 4$ & 48,61 & 26,92 & 11,76 & 3,93 & 1,17 & 0,23 & 1,61 & 1,36 & 1,09 & 1,76 & $|, 8|$ & 0,18 \\
\hline ADI & 50,06 & 23,02 & 9,40 & 5,46 & I,98 & 0,22 & 1,76 & 1,04 & 0,03 & 5,68 & 2,17 & 0,22 \\
\hline AD2 & 47,67 & 22,76 & 9,81 & 6,41 & 1,97 & 0,22 & I,7I & 1,06 & 0,03 & 6,89 & 2,09 & 0,24 \\
\hline AD3 & 45,10 & 22,48 & 10,27 & 7,43 & 1,95 & 0,22 & 1,65 & $\mathrm{I}, 08$ & 0,03 & 8,19 & 2,01 & 0,27 \\
\hline
\end{tabular}

$\mathrm{B}=$ Basicidade $; \mathrm{C} z=$ cinzas .
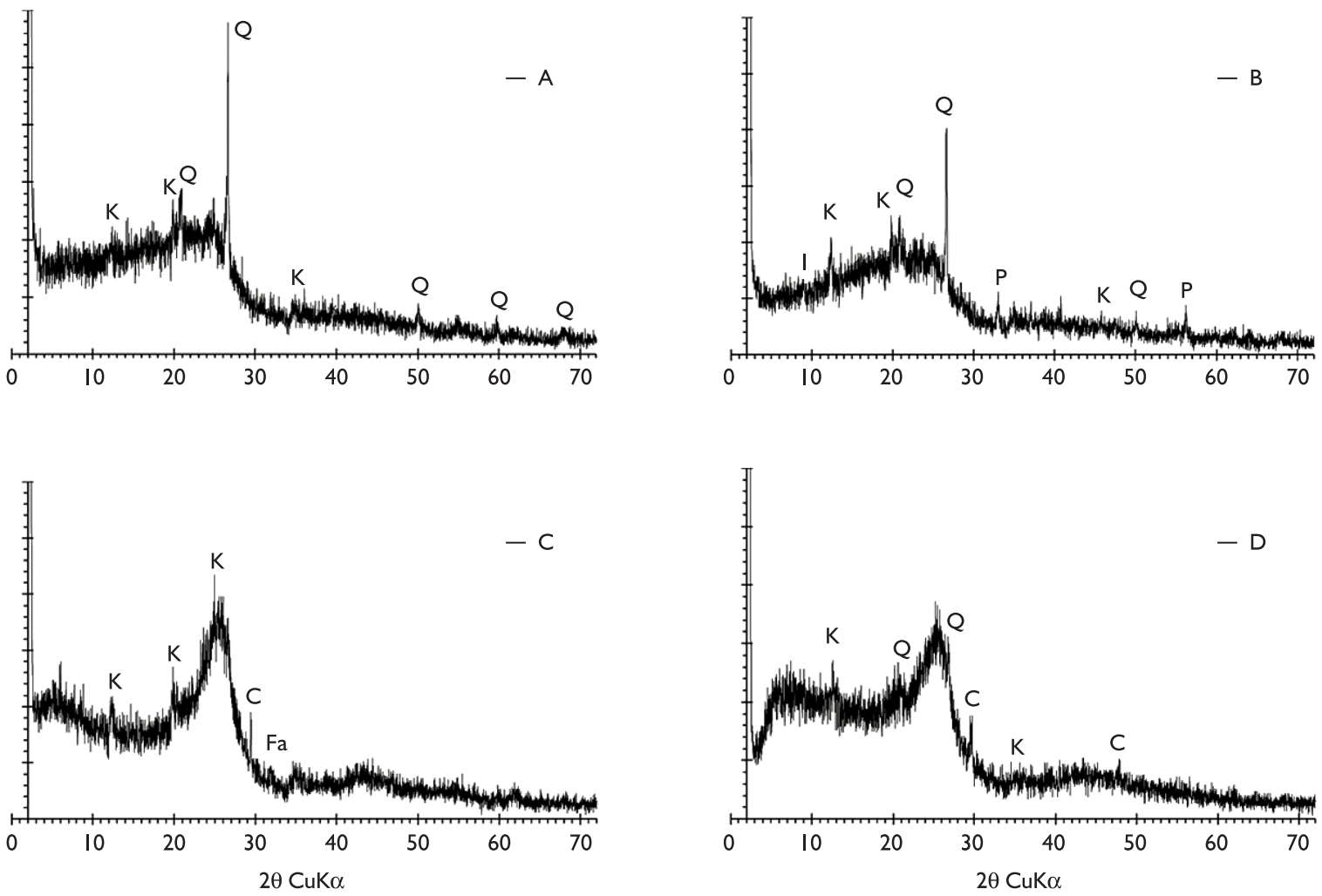

Figura 2. Difratogramas dos carvões $A, B, C$, D. Nas figuras: $\mathrm{C}=$ calcita $\left[\mathrm{CaCO}_{3}\right] ; \mathrm{Fa}=$ fluorapatita $\left[\mathrm{Ca}_{5}\left(\mathrm{PO}_{4}\right)_{3} \mathrm{~F}\right] ; \mathrm{K}=\operatorname{caolinita}\left[\mathrm{Al}_{2} \mathrm{Si}_{2} \mathrm{O}_{5}(\mathrm{OH})_{4}\right]$; $\mathrm{I}=$ ilita $\left[\mathrm{K}_{0.5}(\mathrm{Al}, \mathrm{Fe}, \mathrm{Mg})_{3}(\mathrm{Si}, \mathrm{Al})_{4} \mathrm{O}_{10}(\mathrm{OH})_{2}\right] ; \mathrm{P}=$ pirita $\left[\mathrm{FeS}_{2}\right] ; \mathrm{Q}=$ quartzo $\left[\mathrm{SiO}_{2}\right]$. 


\subsubsection{Principais fases minerais nas cinzas dos carvões e misturas}

A Figura 3 mostra os difratogramas das cinzas dos carvões. A metacaolinita, fase mineral mais importante nas cinzas, é amorfa e, portanto, dificulta a análise de fases com menores concentrações. As fases cristalinas principais nas cinzas dos carvões são quartzo, anidrita, hematita, ilita e sulfato de alumínio hidratado. Nas cinzas "A" também aparece $\circ$ plagioclásio, porém $\circ$ que mais se distingue nestas cinzas é a mais alta intensidade dos picos do quartzo, indicado pelo teor mais elevado de sílica da análise química e que vai originar a razão sílica/alumina mais alta e a basicidade mais baixa. Nas cinzas "B", o pico principal do quartzo é um pouco menos intenso, e a hematita derivada da pirita é uma fase cristalina importante, de acordo com - mais alto teor de Fe encontrado nessa amostra. Nas cinzas "C", destacam-se uma menor intensidade do pico do quartzo, o não aparecimento da ilita e a presença de fases contendo $\mathrm{Ca}$, anidrita (da reação do $\mathrm{S}$ da pirita com a calcita) e fluorapatita, cuja importância está relacionada ao teor de $\mathrm{P}$ (mais alto) desta amostra. A baixa intensidade dos picos de todas as fases cristalinas presentes nas cinzas "C" está relacionada à maior quantidade de fases amorfas, principalmente metacaolinita. As cinzas "D" são caracte- rizadas por uma grande intensidade do pico principal da anidrita, em concordância com os mais altos teores de $\mathrm{CaO}$ e $\mathrm{SO}_{3}$ na análise química. $\mathrm{O}$ aparecimento de sulfato de alumínio hidratado nas cinzas não tem uma origem definida e deve ser melhor investigada.

$A$ análise qualitativa por difração de raios $X$ não se mostrou uma boa metodologia para avaliar as cinzas das misturas de carvões, porque as fases cristalinas principais são as mesmas (quartzo, hematita, anidrita, ilita e sulfato de alumínio) e existe pouca variação na análise química das misturas selecionadas. A diferença mais importante entre os dois tipos de misturas é a presença de mais picos de anidrita e sua maior intensidade nas misturas "AD", de acordo com os mais altos teores de $\mathrm{CaO}$ e $\mathrm{SO}_{3}$ encontrados nessas misturas.

\subsubsection{Principais fases minerais após aquecimento das cinzas a $1.240^{\circ} \mathrm{C}$}

Nos testes a $1.240^{\circ} \mathrm{C}$, resfriados ao ar, as cinzas "C" e principalmente as cinzas " $D$ " e das misturas "AD", apresentaram visualmente fase líquida (fase sólida colada no fundo do cadinho). A presença de fase líquida amorfa interfere nos resultados de DRX, impossibilitando a identificação de fases de menores concentrações.
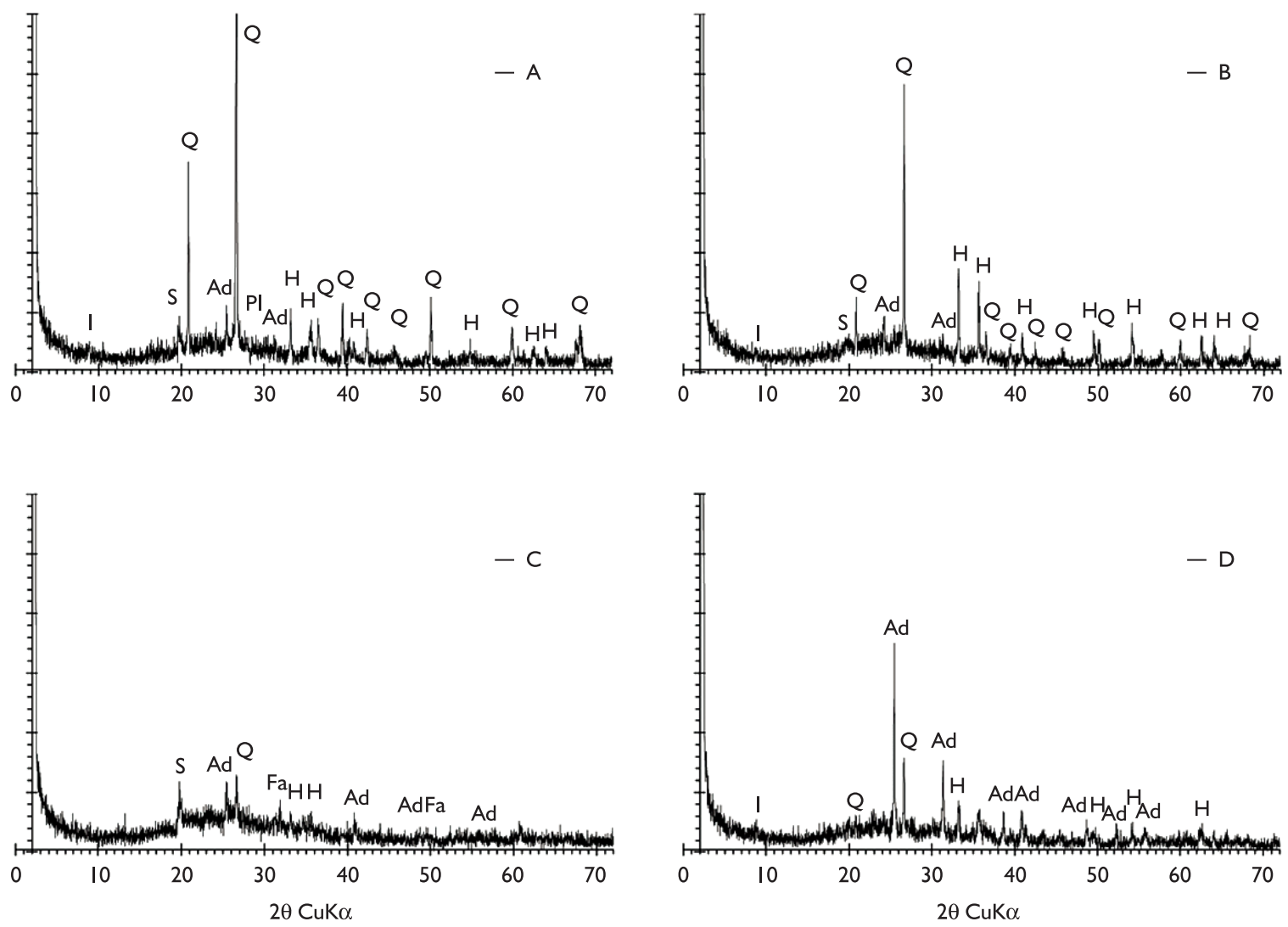

Figura 3. Difratogramas das cinzas $A, B, C, D$. Nas figuras: $A d=$ anidrita $\left[\mathrm{CaSO}_{4}\right] ; \mathrm{F}=$ feldspato de potássio $\left[\mathrm{KAISi}_{3} \mathrm{O}_{8}\right] ; \mathrm{Fa}=$ fluorapatita $\left[\mathrm{Ca}_{5}\left(\mathrm{PO}_{4}\right)_{3} \mathrm{~F}\right] ; \mathrm{H}=$ hematita $\left[\mathrm{Fe}_{2} \mathrm{O}_{3}\right] ; \mathrm{Q}=$ quartzo $\left[\mathrm{SiO}_{2}\right] ; \mathrm{I}=$ ilita $\left[(\mathrm{Na}, \mathrm{K})_{1-x}(\mathrm{Al}, \mathrm{Fe}, \mathrm{Mg})_{2}(\mathrm{Si}, \mathrm{Al})_{4} \mathrm{O}_{10}(\mathrm{OH})_{2}\right] ; \mathrm{S}=$ sulfato de alumínio hidratado $\left[\mathrm{Al}_{2}\left(\mathrm{SO}_{4}\right) \cdot 16 \mathrm{H}_{2} \mathrm{O}\right]$. 
A Figura 4 mostra os difratogramas das amostras de cinzas dos carvões e das misturas sinterizadas a $1.240^{\circ} \mathrm{C}$. Os resultados de DRX mostram que as fases cristalinas mais importantes nas cinzas "A" e "B" são mulita, quartzo e hematita. Nas cinzas " $C$ ", observa-se a mulita como fase majoritária, hematita e anortita, enquanto que nas cinzas "D", a anortita como fase majoritária, hematita e magnetita. Ressalta-se que nas cinzas "C" e "D", - quartzo não aparece. A mulita é formada a partir dos argilominerais, principalmente da metacaolinita, enquanto que a anortita é formada principalmente na reação da mulita com o $\mathrm{CaO}$ liberado na decomposição da anidrita $\left(950^{\circ} \mathrm{C}-1.200^{\circ} \mathrm{C}\right) .^{(14)}$ Nos difratogramas das cinzas da série de misturas "ABC", aquecidas a $1.240^{\circ} \mathrm{C}$, verifica-se que as principais fases cristalinas presentes são mulita, quartzo e hematita. Os difratogramas das cinzas "AD" sinterizadas a $1.240^{\circ} \mathrm{C}$ mostram que as fases mais importantes são anortita, quartzo e hematita. Portanto, a principal diferença entre as misturas " $A B C$ " e "AD" é que as primeiras têm como fase majoritária em temperaturas altas a mulita e as últimas, a anortita.

\subsection{Testes de Fusibilidade de Cinzas}

Os testes de fusibilidades de cinzas são testes dinâmicos, onde com o aumento da temperatura ocorrem vários processos como sinterização das partículas de cinzas, difusão de espécies químicas, formação de uma fase de escória líquida a partir de partículas de baixo ponto de fusão; decomposição de alguns compostos com liberação de gás; dissolução de partículas sólidas no líquido formado. Como resultado do aumento de fase líquida com o aumento da temperatura, o cone de cinzas se deforma, muda para forma esférica e para semi-esférica antes de fluir. Entre os fatores que afetam a cinética de dissolução das cinzas estão a composição mineralógica e o tamanho da partícula. ${ }^{(15)}$

\subsection{Testes de fusibilidade das cinzas dos carvões}

Os testes de fusibilidade podem ser divididos em dois intervalos: o intervalo de amolecimento, entre a temperatura de deformação inicial (IT) e a de semi-esfera $(\mathrm{HT})$ e o intervalo de fusão, entre a temperatura de semi-esfera $(\mathrm{HT})$ e a de fluidez (FT).
A Tabela 4 apresenta os resultados dos testes de fusibilidade das cinzas dos carvões e das misturas de carvões. Observa-se que as cinzas dos carvões "A" e "B" iniciam o processo de amolecimento em temperaturas mais altas que as cinzas "C" e " $D$ ". No entanto, as cinzas "B" e " $D$ " apresentam temperaturas de fluidez mais baixas. As cinzas A, preparadas a $1.240^{\circ} \mathrm{C}$ foram fáceis de moer para a $D R X$, indicando uma menor quantidade de líquido formado até esta temperatura. Segundo Vassilev et al. (14), teores altos de quartzo e mulita tendem a elevar as temperaturas de fusibilidade. $O$ amolecimento começa quando existe extensiva quantidade líquido, com composição próxima a ferrocordierita, ${ }^{(16)}$ conforme diagrama de equilíbrio $\mathrm{SiO}_{2}-\mathrm{Al}_{2} \mathrm{O}_{3}-\mathrm{FeO}\left(\mathrm{Fe}_{2} \mathrm{O}_{3}\right)$. A maior quantidade de quartzo e a difusão de cátions de $\mathrm{Fe}$ e de outros cátions básicos do líquido para a mulita levam a temperatura FT para $1.434^{\circ} \mathrm{C}$. Nas cinzas "B", mais ricas em hematita, provavelmente a formação de mais espinélios $\left(\mathrm{Fe}_{3} \mathrm{O}_{4}, \mathrm{AlFe}_{2} \mathrm{O}_{4}, \mathrm{MgFe}_{2} \mathrm{O}_{4}\right)$ retarda a formação de líquido(14) e $\mathrm{O}$ amolecimento inicia em temperatura mais alta. No entanto, a maior proporção de hematita nas cinzas resulta num intervalo de amolecimento e fusão menor $\left(32^{\circ} \mathrm{C}\right)$, e numa FT de $1405^{\circ} \mathrm{C}$. Nas cinzas do carvão "C", as fases cristalinas presentes a $1.240^{\circ} \mathrm{C}$ são mulita, anortita e hematita. A temperatura IT mais baixa dessas cinzas indica maior proporção de fases de mais baixo ponto de fusão, porém o teor maior de mulita e menor de quartzo dessa amostra estende o intervalo de amolecimento e fusão $\left(281^{\circ} \mathrm{C}\right)$ até $1.514^{\circ} \mathrm{C}$. Conforme Vassilev et al., ${ }^{(14)}$ a mulita, fase mais refratária do sistema, eleva mais as temperaturas $\mathrm{HT}$ e FT do que o quartzo. As cinzas do carvão "D”, mais ricas em Ca e S, elementos fluxantes, apresentam a $1.240^{\circ} \mathrm{C}$ como fases cristalinas, a anortita, em maior proporção, e hematita. $\mathrm{O}$ quartzo no teste de $1240^{\circ} \mathrm{C}$ já foi assimilado no líquido. A difusão dos cátions de $\mathrm{Fe}$ e de outros cátions básicos (provavelmente na fase líquida) na rede da anortita e/ou sua dissolução no líquido reduzem o intervalo de amolecimento e fusão $\left(35^{\circ} \mathrm{C}\right)$ e a $\mathrm{FT}$ para $1.339^{\circ} \mathrm{C}$.

\subsubsection{Testes de fusibilidade das cinzas das misturas}

Observa-se também na Tabela 4 que as temperaturas de fusibilidade das cinzas " $\mathrm{ABCl}$ " têm um comportamento de fusibilidade mais próximo das cinzas "A", porém com um intervalo de amolecimento e fusão aumentado em $29^{\circ} \mathrm{C}$, resultando numa $\mathrm{FT}$ maior, devido

Tabela 4. Temperaturas de fusibilidade das cinzas dos carvões e das misturas

\begin{tabular}{|c|c|c|c|c|c|c|c|c|c|c|c|}
\hline Amostra & $\mathbf{A}$ & B & C & D & $\mathrm{ABCl}$ & $A B C 2$ & ABC3 & ABC4 & ADI & AD2 & AD3 \\
\hline IT $\left({ }^{\circ} \mathrm{C}\right)$ & 1342 & 1373 & 1233 & 1279 & $133 \mid$ & 1387 & 1427 & 1423 & 1273 & 1259 & 1275 \\
\hline ST $\left({ }^{\circ} \mathrm{C}\right)$ & 1362 & 1382 & $133 \mid$ & 1293 & 1369 & 1415 & 1450 & 1462 & 1279 & 1262 & 1286 \\
\hline $\mathrm{HT}\left({ }^{\circ} \mathrm{C}\right)$ & 1400 & 1392 & 1469 & 1314 & $|4| \mid$ & $|44|$ & 1480 & 1484 & 1310 & 1300 & 1311 \\
\hline $\mathrm{FT}\left({ }^{\circ} \mathrm{C}\right)$ & 1434 & 1405 & 1514 & 1339 & 1462 & 1450 & 1506 & 1517 & 1366 & 1337 & $135 \mid$ \\
\hline
\end{tabular}



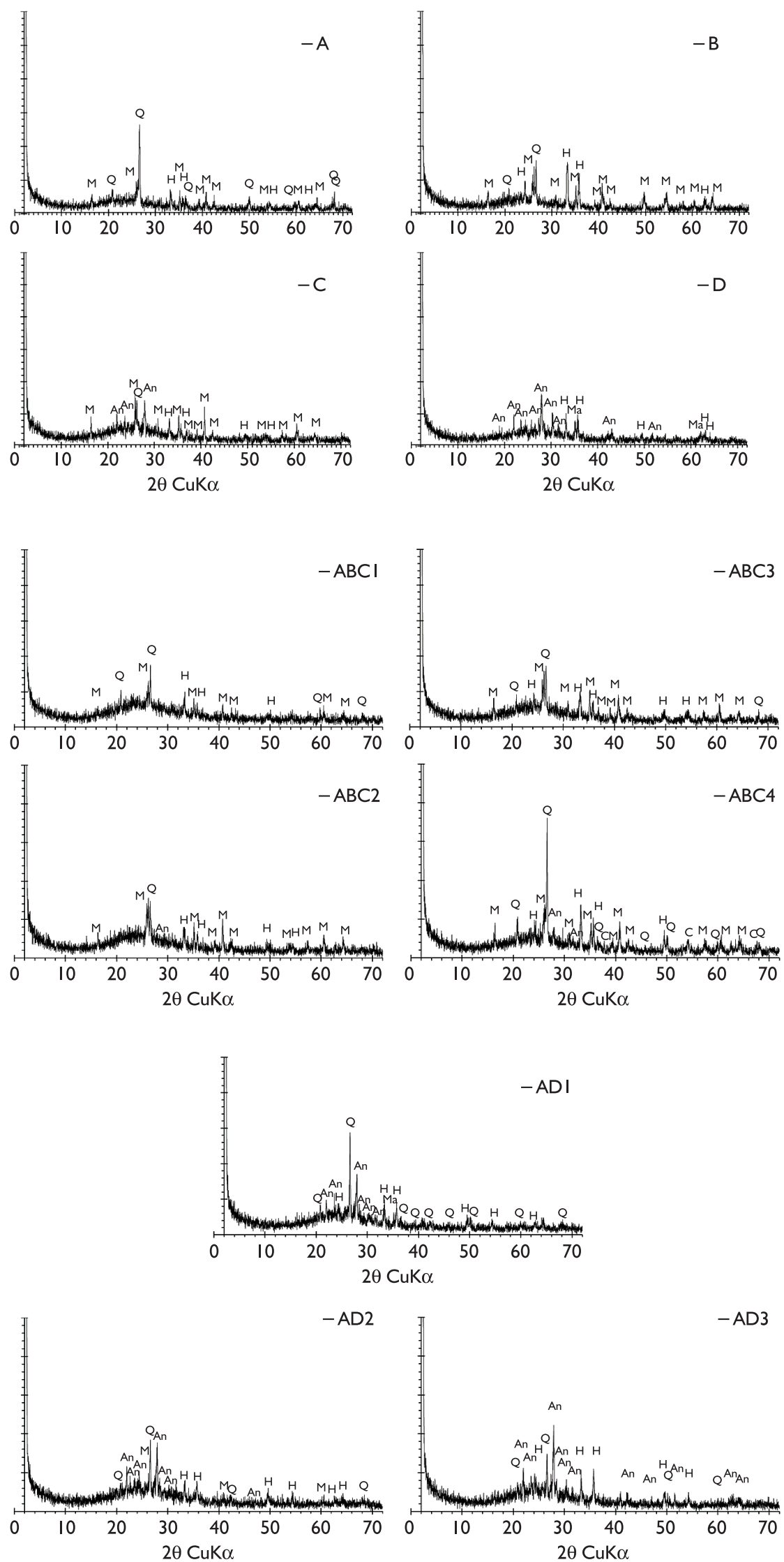

Figura 4. Difratogramas das cinzas dos carvões (A, B, C, D) e das séries de misturas (ABC e AD) aquecidas a $1240^{\circ} \mathrm{C}$ e resfriadas ao ar. Nas figuras: $\mathrm{An}=$ anortita $\left[\mathrm{CaAl}_{2} \mathrm{Si}_{2} \mathrm{O}_{8}\right] ; \mathrm{C}=\mathrm{cal}[\mathrm{CaO}] ; \mathrm{H}=$ hematita[ $\left[\mathrm{Fe}_{2} \mathrm{O}_{3}\right] ; \mathrm{Q}=$ quartzo[ $\left[\mathrm{SiO}_{2}\right] ; \mathrm{M}=\operatorname{mulita}\left[\mathrm{Al}_{4,57} \mathrm{Si}_{1,41} \mathrm{O}_{9,7}\right] ; \mathrm{Ma}=\operatorname{magnetita}\left[\mathrm{Fe}_{3} \mathrm{O}_{4}\right]$. 
principalmente às cinzas " $\mathrm{C}$ ", mais ricas em mulita. Nas cinzas "ABC2", percebe-se que há um aumento da influência das cinzas "B" (com mais hematita e menos quartzo que "A") em função do aumento das temperaturas IT, ST e HT e uma redução do intervalo de fusão em relação a "ABCI", mas com FTs iguais. Nas cinzas "ABC3" e "ABC4", verificam-se a maior influência da mulita e menor do quartzo nas temperaturas de fusibilidade, com a elevação das temperaturas ST, HT e FT. É importante notar que a mudança na análise química das amostras " $A B C$ " é relativamente pequena (Tabela 3) e, portanto, a composição mineralógica é mais importante na fusibilidade das cinzas. As temperaturas de fusibilidade dessas misturas não são aditivas em relação àquelas dos carvões componentes, uma vez que a FT da mistura "ABC4", por exemplo, é praticamente igual a das cinzas " $C$ ".

Os resultados de fusibilidade das cinzas das misturas "AD", na Tabela 4, mostram temperaturas mais próximas das cinzas do carvão " $D$ " do que "A", sendo que as cinzas da mistura "AD2" apresentam IT, ST, HT menores que " $D$ " e a mesma FT. As cinzas "ADI" e "AD3" apresentam o mesmo intervalo de amolecimento e pequena diferença $\left(15^{\circ} \mathrm{C}\right)$ no intervalo de fusão, enquanto que a amostra "AD2" apresenta as menores temperaturas de fusibilidade. Isto indica novamente a não aditividade das temperaturas de fusibilidade das misturas em relação àquelas das cinzas dos carvões componentes. As cinzas "AD" em I. $240^{\circ} \mathrm{C}$ têm como fases principais a anortita, hematita e quartzo em proporções diferentes, enquanto que as cinzas do carvão "A" apresentam mulita, hematita e quartzo e as cinzas " $D$ ", anortita e hematita. Isto significa que as quantidades de $\mathrm{Ca}$ e Fe presentes nas cinzas " $D$ " são suficientes para transformar a mulita presente nas cinzas "A" em anortita, nas proporções de carvões testadas, levando as misturas para regiões de temperaturas liquidus mais baixas.

Comparando as misturas "AD" e "ABC", observa-se que as primeiras se tornam líquidas em temperaturas inferiores devido à formação e maior proporção de anortita e as últimas fundem em temperaturas mais altas devido à maior proporção de mulita.

\subsection{Testes de viscosidade de cinzas}

A viscosidade é a medida da resistência de um fluido ao escoamento. Nos silicatos líquidos, a estrutura é fortemente ligada por pontes de oxigênio e são necessários cátions básicos (modificadores) para provocar a quebra das cadeias de ânions e reduzir a viscosidade. ${ }^{(10)}$

\subsection{Viscosidade das cinzas dos carvões}

As curvas de viscosidade das cinzas dos carvões em função da temperatura, na Figura 5, mostram que as escórias líquidas das cinzas dos carvões têm diferentes tipos de comportamento. As cinzas "A", com a mais baixa basicidade $(B=0,14)$, apresentam uma escória longa e viscosa até $1.580^{\circ} \mathrm{C}$, devido à grande quantidade de sílica presente, sem o teor necessário de cátions básicos para romper totalmente a cadeia de tetraedros da sílica. $\mathrm{Na}$ temperatura de fluidez, a viscosidade é de 1 . II 5 poise. As cinzas "B" têm uma escória de baixa viscosidade, menor que 250 poise, a partir de $1.419^{\circ} \mathrm{C}$, em função da maior quantidade de óxidos básicos, em comparação à amostra "A". As cinzas "C" mostram viscosidades baixas somente acima de $1.510^{\circ} \mathrm{C}$. Abaixo dessa temperatura com 0 início da cristalização da mulita aumenta rapidamente a viscosidade. As cinzas " $D$ " apresentam as mais baixas viscosidades entre as cinzas no intervalo de temperatura testado (3-57poise). Isto se deve à alta concentração de óxidos básicos presentes, para uma razão sílica/alumina relativamente baixa.

Comparando as cinzas dos carvões a $1500^{\circ} \mathrm{C}$, verifica-se na Figura 5 que as escórias das cinzas "A" e "C" têm alta viscosidade em função dos teores mais altos de $\mathrm{Si}$ e $\mathrm{Al}$ e de teores insuficientes de $\mathrm{Fe}, \mathrm{Ca}$ e demais elementos alcalinos e alcalinos-terrosos, enquanto que as escórias "B" e "D", devido principalmente aos maiores teores desses elementos tem viscosidade baixa.

\subsubsection{Viscosidade das cinzas das misturas de carvões}

A Figura 6 apresenta as curvas de viscosidade das cinzas das misturas "ABC" vs. temperatura, comparadas àquelas dos carvões componentes. A $1.500^{\circ} \mathrm{C}$ essas escórias são viscosas, devido ao mais alto teor de Al. A mistura "ABC4" têm curva de viscosidade em função da temperatura semelhante àquela das cinzas "C". Entretanto, as viscosidades de "C" são mais baixas entre $1.550^{\circ} \mathrm{C}$ e $1.600^{\circ} \mathrm{C}$ devido à maior basicidade. $\mathrm{Em} 1.600^{\circ} \mathrm{C}$ todas as misturas têm viscosidade menor que 100 poise, porém as misturas " $\mathrm{ABCl}$ " e "ABC2" apresentam viscosidades superiores a "ABC3" e "ABC4" em função do maior teor de sílica.

As curvas de viscosidade em função da temperatura das misturas "AD", na Figura 7, mostram que são escórias longas, com comportamentos intermediários às cinzas dos carvões "A" e "D". A queda da viscosidade de "ADI" para "AD3" se deve ao aumento da basicidade e ao decréscimo da razão sílica/alumina.

Comparando a viscosidade das escórias "ABC" e "AD" a $1.500^{\circ} \mathrm{C}$, as primeiras apresentam viscosidade mais alta em função dos maiores teores de Al e da baixa basicidade, enquanto que as últimas mostram viscosidades mais baixas, devido principalmente à maior basicidade em cinzas com razões sílica/alumina mais altas. 


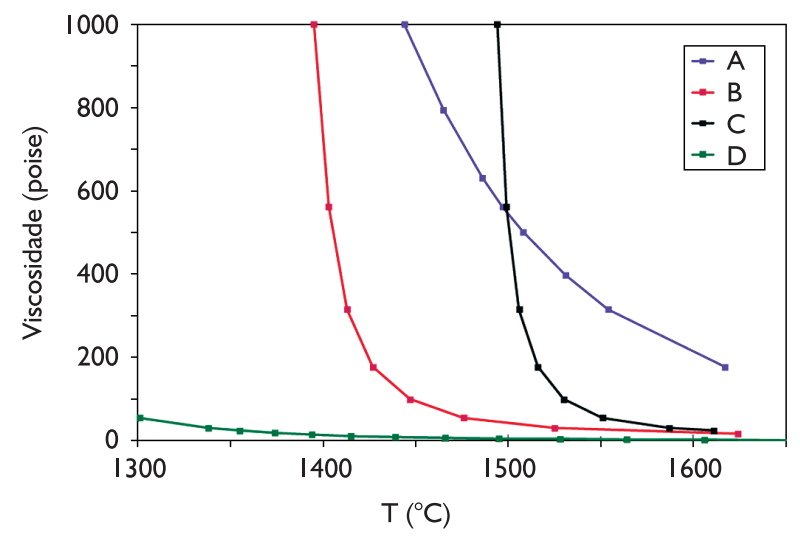

Figura 5. Viscosidade das cinzas dos carvões em função da temperatura.

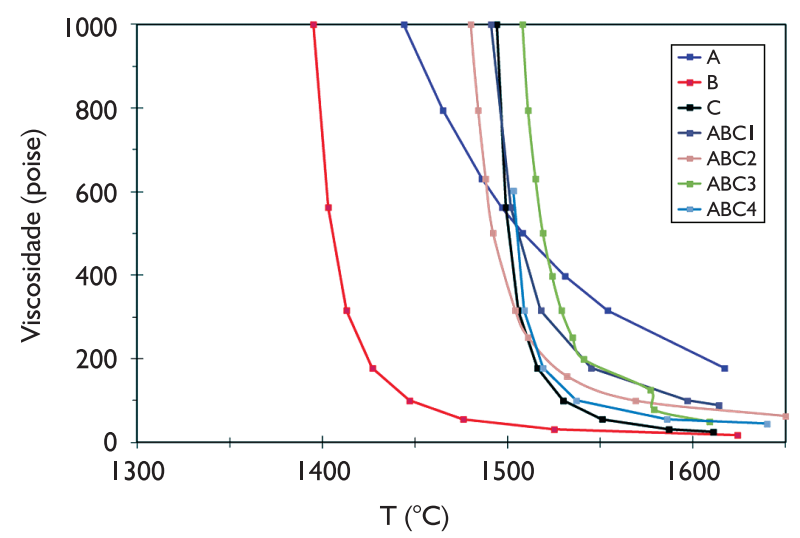

Figura 6. Viscosidade das cinzas das misturas $A B C$ comparadas as cinzas A, B e C.

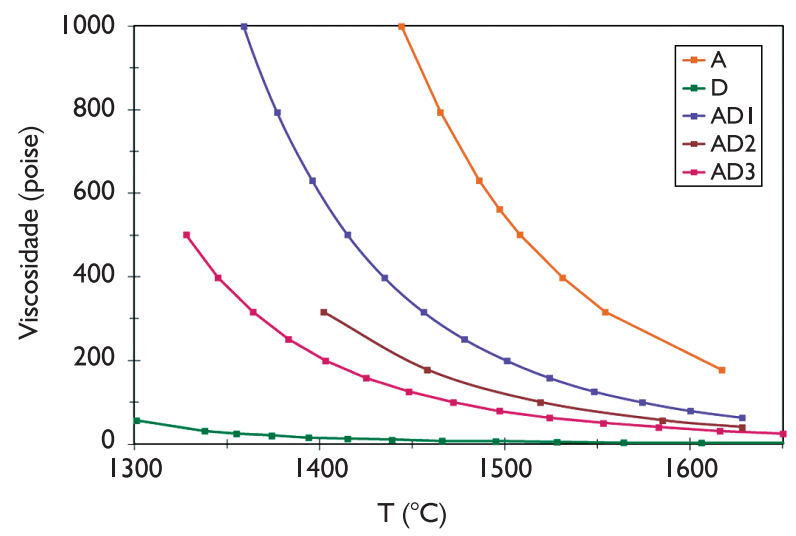

Figura 7. Viscosidade das cinzas das misturas $A D$ comparadas às cinzas A e D.

\section{CONCLUSÕES}

De acordo com os resultados, cinzas com teores mais altos de $\mathrm{Si}$ e Al apresentam temperatura de fluidez e viscosidade altas a $1.500^{\circ} \mathrm{C}$, enquanto que aquelas com teores importantes em $\mathrm{Fe}$, Ca e $\mathrm{S}$ têm temperaturas de fluidez e viscosidade mais baixa.
No comportamento de fusibilidade das cinzas, pode-se concluir que:

- as cinzas do carvão "C" mostram o mais longo intervalo de fusibilidade e a maior temperatura de fluidez entre os carvões, em função da maior proporção de mulita, derivada de argilominerais, principalmente caolinita;

- as cinzas "A", mais ricas em $\mathrm{Si}$, apresentam o segundo intervalo maior de fusibilidade, que se deve a principalmente à presença de mulita $\mathrm{e}$ quartzo, em teor mais elevado;

- as cinzas do carvão "B" têm o menor intervalo de fusibilidade, em função dos teores de quartzo e mulita, e da proporção relativamente alta de $\mathrm{Fe}$, hematita, oriunda principalmente da pirita;

- as cinzas do carvão "D", com teores de $\mathrm{Fe}, \mathrm{Ca}$ e $S$ relativamente altos, apresentam a menor temperatura de fluidez, devido à formação da fase anortita, resultante da reação da anidrita e mulita, sendo a anidrita originada na reação dos minerais pirita e calcita;

- as cinzas das misturas " $A B C$ " têm temperaturas de fluidez mais altas que as misturas "AD" devido principalmente à grande proporção de mulita; e

- as cinzas das misturas "AD" apresentam temperaturas de fusibilidades mais baixas, em função da formação de maiores proporções de anortita.

No comportamento de viscosidade das cinzas com a temperatura, conclui-se que:

- as cinzas "A" formam uma escória longa e viscosa até $1.580^{\circ} \mathrm{C}$ devido à grande quantidade de sílica presente e a baixa basicidade;

- a escória "B" tem uma viscosidade baixa a partir de $1.420^{\circ} \mathrm{C}$, em função da maior quantidade de óxidos básicos presentes e da menor relação sílica/alumina, comparada a amostra $\mathrm{A}$;

- a escória "C" mostram viscosidades baixas somente acima de $1.510^{\circ} \mathrm{C}$. Abaixo dessa temperatura, a viscosidade aumenta rapidamente devido à cristalização da mulita;

- a escória "D" apresenta viscosidade bem baixa em todo o intervalo de temperatura medido, em função da mais alta basicidade e de uma relativamente baixa razão sílica /alumina;

- as misturas "ABC" têm uma alta viscosidade a $1.500^{\circ} \mathrm{C}$ devido ao alto teor de $\mathrm{Al}$ e a uma baixa basicidade; e

- as misturas "AD" mostram uma baixa viscosidade a $1.500^{\circ} \mathrm{C}$ em função da maior basicidade de cinzas com relativamente altas razões sílica/alumina.

\section{Agradecimentos}

Os autores agradecem a Arcelormittal Tubarão, a Global R\&D Arcelormittal, a Capes e CNPq - Rede de Pesquisas em Carvão Mineral. 


\section{REFERÊNCIAS}

I DEFENDI, G. A. et al. Development of knowledge on combustion and ash behavior of pulverized coals injected in ArcelorMittal Tubarão blast furnaces. Revue de Metallurgie - CIT, n. 7-8, p. 346-55, juil.août 2008.

2 ICHIDA, M. et al. Behavior of pulverized coal ash and physical property of dripping slag under high pulverized coal injection operation. ISI International, v. 4I, n. 4, p. 325-32, 200 I.

3 WIKSTRÖM, J.-O., e al. Improuved slag formation in the blast furnace by co-injection of basic fluxes, together with pulverized coal, through the tuyeres. In: INTERNATIONAL BF LOWERZONE SYMPOSIUM, 2002, Wollongong. Proceedings... Carlton Victoria: Australasian Institute of Mining and Metallurgy, 2002. p. I8-I-I8-I4.

4 BAGATINI, M. C. et al. Estudo de fusibilidade das cinzas de carvões em função da composição química e mineralógica. Tecnologia em Metalurgia e Materiais, v. 3, n. 4, p. 52-58, abr.-jun. 2007. http://dx.doi.org/l0.4322/ tmm.00304009

5 BAGATINI, M. C. et al. The behaviour of coal ashes for $\mathrm{PCl}$ at high temperatures in relation to their chemical and mineralogical composition - experimental and computational analysis. Ironmaking and Steelmaking, v. 36, n. 8, p. 583-589, Nov. 2009. http://dx.doi.org/I0. I I 79/030192309XI 2492910938050

6 ASTM INTERNATIONAL. ASTM D7582-10. Standard test methods for proximate analysis of coal and coke by macro thermogravimetric analysis. West Conshohocken, 2010.

7 ASTM INTERNATIONAL. ASTM D4239-10. Standard test methods for sulfur in the analysis sample of coal and coke using high-temperature tube furnace combustion methods. West Conshohocken, 2010.

8 ASTM INTERNATIONAL. ASTM D4326-04. Standard test method for major and minor elements in coal and coke ash by $\mathrm{x}$-ray fluorescence. West Conshohocken, 2004.

9 ASTM INTERNATIONAL. ASTM D50 I6-08e I. Standard test method for total sulfur in coal and coke combustion residues using a high-temperature tube furnace combustion method with infrared absorption. West Conshohocken, 2008.

IO VARGAS, S.; FRANDSEN, F. J.; DAM-JOHANSEN, K. Rheological properties of high-temperature melts of coal ashes and other silicates. Progress in Energy and Combustion Science, v. 27, n. 3, p. 237-429, 200 I. http://dx.doi. org/I0.1016/S0360-1285(00)00023-X

I I ASTM INTERNATIONAL. ASTM D/857-04. Standard test method for fusibility of coal and coke ash. West Conshohocken, 2004.

I 2 ASTM INTERNATIONAL. ASTM C-965-96(2007). Standard practice for measuring viscosity of glass above the softening point. West Conshohocken, 2007.

I 3 DEUTSCHES INSTITUT FÜR NORMUNG. DIN 5 / 730-1998-04. Testing solid fuels. Determination of fusibility of fuel ash. Berlin, 1998.

14 VASSILEV, S. V. et al. Influence of mineral and chemical composition of coal ashes on their fusibility. Fuel Processing Technology, v. 45, n. I, p. 27-5I,Oct. 1995. http://dx.doi.org/I0.1016/0378-3820(95)00032-3

I 5 JAK, E. Prediction of coal ash fusion temperatures with the $F^{*} A^{*} C^{*} T^{*}$ thermo-dynamics computer package. Fuel, v. 8I, n. I3, p. 1655-68, Aug. 2002. http://dx.doi.org/l0.1016/S00 I6-236I(02)0009 I-I

16 WALL, T. F. et al. Coal ash fusion temperature-New characterization techniques and implications for slagging and fouling. Progress in Energy and Combustion Science, v. 24, n. 4, p. 345-53, 1998. http://dx.doi.org/10.1016/S03601285(98)000 10-0

Recebido em: 16/10/2011

Aceito em: 20/04/2012 